\title{
Improved Variance Reduced Monte-Carlo Simulation of in-the-Money Options
}

\author{
Armin Müller \\ FernUniversität in Hagen, Lehrstuhl für angewandte Statistik und Methoden der empirischen Sozialforschung, \\ Hagen, Germany \\ Email: armin.mueller@fernuni-hagen.de
}

Received 29 May 2015; accepted 30 July 2016; published 2 August 2016

Copyright (C) 2016 by author and Scientific Research Publishing Inc.

This work is licensed under the Creative Commons Attribution International License (CC BY).

http://creativecommons.org/licenses/by/4.0/

c) (i) Open Access

\section{Abstract}

Pricing derivatives with Monte-Carlo simulations involve standard errors that typically decrease at a rate proportional to $1 / \sqrt{N}$ where $N$ is the sample size. Several approaches have been discussed to reduce the empirical variance for a given sample size. This article analyzes the joint application of the put-call-parity approach and importance sampling to variance reduced option pricing. For this purpose, we examine non-path-dependent and path-dependent options. For European options, we observe dramatic variance reduction, especially for in-the-money options. Also for arithmetic Asian options, a significant variance reduction is achieved.

\section{Keywords}

\section{Monte-Carlo Simulation, Variance Reduction, Importance Sampling, Put-Call-Parity, Asian Option}

\section{Introduction}

Monte-Carlo simulations are frequently used to estimate prices of financial products for which no analytical formulae exist, e.g., several path-dependent options. A difficulty related to this approach is that the empirical variance of estimators decreases slowly, typically at a rate $\propto 1 / N$ where $N$ is the sample size. Several techniques have been discussed to reduce the empirical variance for a given sample size $N$ [1]. Importance sampling turns out to be a particularly effective variance reduction technique [2]-[5].

An alternative approach to variance reduction at least for in-the-money options is the application of the put-call-parity: instead of simulating an in-the-money call price, the corresponding out-of-the-money put price can be simulated. Then, the call price can be calculated from the put-call-parity yielding a variance reduced estimator [6] [7].

This analysis will investigate how the joint application of the put-call-parity and importance sampling can 
lead to significant variance reduction in the simulation of Monte-Carlo estimators. The article is organized as follows: in Section 2 the method of importance sampling is introduced. Section 3 explains how put-call-parities can be employed for variance reduction. Next, the combination of the two approaches is introduced in Section 4. Numerical results of the achieved variance reductions are presented in Section 5 for both non-path-dependent and path-dependent options. A conclusion follows in Section 6.

\section{Importance Sampling}

Foundations of Importance Sampling This section introduces the concept of importance sampling, which serves to reduce the empirical variance of estimators.

The expectation value of a non-negative function $h: \mathbb{R}^{d} \rightarrow \mathbb{R}_{0}^{+}, X \rightarrow h(X)$ of a random variable $X$ with probability density $p$ is calculated as

$$
\alpha=\mathbf{E}_{p}[h(X)] \equiv \int h(x) p(x) \mathrm{d} x .
$$

An estimator of $\alpha$ with $N$ i.i.d. realizations $X_{1}, \cdots, X_{N}$ of the random variable $X$ is

$$
\hat{\alpha}_{p}=\frac{1}{N} \sum_{i=1}^{N} h\left(X_{i}\right)
$$

With any other probability density $p^{\prime}, \alpha$ can be rewritten as

$$
\alpha=\int h(x) p(x) \mathrm{d} x=\int h(x) \frac{p(x)}{p^{\prime}(x)} p^{\prime}(x) \mathrm{d} x \equiv \mathbf{E}_{p^{\prime}}\left[h(X) \frac{p(X)}{p^{\prime}(X)}\right] .
$$

The fraction $p / p^{\prime}$ is usually called "Radon-Nikodým derivative".

An unbiased estimator of this expression is

$$
\hat{\alpha}_{p^{\prime}}=\frac{1}{n} \sum_{i=1}^{n} h\left(X_{i}\right) \frac{p\left(X_{i}\right)}{p^{\prime}\left(X_{i}\right)} .
$$

Its variance

$$
\operatorname{Var}_{p^{\prime}}\left[h(X) \frac{p(X)}{p^{\prime}(X)}\right]=\mathbf{E}_{p^{\prime}}\left[\left(h(X) \frac{p(X)}{p^{\prime}(X)}\right)^{2}\right]-\mathbf{E}_{p^{\prime}}\left[\left(h(X) \frac{p(X)}{p^{\prime}(X)}\right)\right]^{2}
$$

vanishes if we choose

$$
p^{\prime}(X) \propto h(X) p(X) .
$$

To obtain a a new probability density this product must be normalized (note that $h \geq 0$ by definition):

$$
p^{\prime}(X)=p^{\text {opt }}(X)=\frac{h(X) p(X)}{\mathbf{E}_{p}[h(X)]} .
$$

For this purpose, the integral

$$
\mathbf{E}_{p}[h(X)]=\int h(x) p(x) \mathrm{d} x
$$

must be calculated, which is the original problem to be solved in Equation (1). However, an approximation of the optimal density can already lead to significant variance reduction [1].

Monte-Carlo simulation of the Feynman-Kac formula Option prices can be calculated as the expectation value of the derivative's discounted pay-off function. In the following, a variance reduced estimator of the resulting Feynman-Kac formula

$$
C\left(S_{t}, t\right)=\mathbf{E}\left[\mathrm{e}^{-\int_{t}^{T} r\left(S_{\tau}, \tau\right) \mathrm{d} \tau} h\left(S_{T}\right) \mid S(t)=S_{t}\right]
$$

will be presented. Note that the Feynman-Kac formula also covers the case of path-dependent options, i.e. the case where $h$ does not only depend on the terminal value of the underlying's price $S_{T}$ but also on other values 
$S_{t}$ with $0<t<T$. Instead of writing $S_{t}$ in Equation (9), one could write $X_{t}$ and define $X_{t}$ as a bivariate random process whose first element is the underlying price $S_{t}$ itself and the second element is the integral of $S_{t}$ with respect to time.

For simplicity, a constant risk-free interest rate $r$ is assumed. For the purpose of numerical calculations, the Feynman-Kac formula can be expressed as

$$
C\left(S_{t}, t\right) \approx \mathrm{e}^{-r(T-t)} \int h\left(S_{n}\right) p\left(S_{n}, \tau_{n} \mid S_{n-1}, \tau_{n-1}\right) \times \cdots \times p\left(S_{1}, \tau_{1} \mid S_{t}, t\right) \mathrm{d} S_{n} \cdots \mathrm{d} S_{1}
$$

using a finite-dimensional approximation on a grid $\tau_{k}=t+k \Delta \tau$ with Euler densities $p\left(S_{i}, \tau_{i} \mid S_{i-1}, \tau_{i-1}\right)$ and $n$ discretization steps (for details see Appendix B in [5]). We set $\tau_{n}=T$ and consequently $S_{n}=S_{T}$.

In accordance with Equation (2), a simple Monte-Carlo estimator of this expression is

$$
\hat{C}(S(t), t)=\frac{1}{N} \sum_{i=1}^{N} \hat{C}_{i}=\mathrm{e}^{-r(T-t)} \frac{1}{N} \sum_{i=1}^{N} h\left(S_{i}(T)\right) .
$$

Importance sampling by adding an additional drift term In 2014, Singer presented a variance reduced Monte-Carlo estimator for Equation (9) which relies on importance sampling by adding an additional drift term to the stochastic differential equation

$$
\mathrm{d} S(t)=f(S(t)) \mathrm{d} t+g(S(t)) \mathrm{d} W(t)
$$

where $f(S(t))$ is a drift parameter, $t$ the time, $g(S(t))$ a diffusion coefficient and $W(t)$ a Wiener process. Here we just present the result, while the lenghty derivation can be found in [5]. Melchior and Öttinger have introduced a similar approach in 1995 [2].

The optimal importance sampling density $p^{\prime}=p^{\text {opt }}$ is chosen as indicated in Equation (7). It can be shown that this density is equivalent to a new stochastic differential equation with additional drift term of the form

$$
\mathrm{d} S=f^{\text {opt }} \mathrm{d} t+g \mathrm{~d} W=\left(f+\frac{g^{2}}{C} \frac{\partial C}{\partial S}\right) \mathrm{d} t+g \mathrm{~d} W .
$$

In the Black-Scholes model with $f=r S$ and $g=\sigma S$, one obtains

$$
\mathrm{d} S=\left(r+\epsilon \sigma^{2}\right) S \mathrm{~d} t+\sigma S \mathrm{~d} W
$$

using the abbreviation

$$
\epsilon=\frac{S}{C} \frac{\partial C}{\partial S}
$$

for the option price elasticity.

Here, $C$ is the Feynman-Kac formula from Equation (9). Again, the problem of Equations (7) and (8) materializes: To describe the optimal stochastic differential equation, knowledge of the unknown quantity $C$ is required.

However, variance reduction can also be achieved by approximating $C$. For this purpose, in our analysis we use the Black-Scholes formula for European options [8]. This formula is the best possible choice when simulating European options in the Black-Scholes model, as it yields the analytically exact value. But also for other models of the underlying price (e.g., the CEV model or models with stochastic volatility) or for other options (e.g., Asian options) it represents a useful approximation.

In the univariate Black-Scholes case that is considered in this article, the Radon-Nikodým derivative for one step on the discretized grid yields

$$
\frac{p}{p^{\mathrm{opt}}}=\exp \left\{-\sigma \epsilon \Delta W-\frac{1}{2} \sigma^{2} \varepsilon^{2} \Delta t\right\} .
$$

Ultimately, for the variance reduced Monte-Carlo estimator with sample size $N, n$ discretization steps and sampling from $p^{\text {opt }}$ one obtains

$$
\hat{C}^{\mathrm{IS}}(S(t), t)=\frac{1}{N} \sum_{i=1}^{N} \hat{C}_{i}^{\mathrm{IS}}=\frac{1}{N} \sum_{i=1}^{N} \exp \left\{-\sum_{k=0}^{n-1}\left[\sigma \epsilon_{i k} \Delta W_{i k}+\frac{1}{2} \sigma^{2} \epsilon_{i k}^{2} \Delta t\right]\right\} \times \mathrm{e}^{-r(T-t)} h\left(S_{i}(T)\right) .
$$


As mentioned, $\epsilon_{i k}$ can be calculated from the Black-Scholes formula [8]. Depending on whether $h$ represents the pay-off function of a call option or a put option, one obtains [9]

$$
\epsilon_{\text {Call,Put }}(S(t), t)=\left(1-\frac{K \mathrm{e}^{-r(T-t)}}{S(t)} \frac{\Phi\left( \pm d_{2}(S(t), t)\right)}{\Phi\left( \pm d_{1}(S(t), t)\right)}\right)^{-1} .
$$

\section{Variance Reduction with Put-Call-Parities for European and Arithmetic Asian Options}

European options Simple non-arbitrage arguments require that for a European call with pay-off function

$$
C_{T}=\left(S_{T}-K\right)^{+}
$$

the following put-call-parity holds true where $C_{0}$ is the initial call price at $t=0$ and $P_{0}$ the put price [10]:

$$
C_{0}=P_{0}+S_{0}-K \mathrm{e}^{-r T}
$$

Arithmetic Asian options Also for arithmetic Asian call options with the pay-off function

$$
C_{T}=\left(\bar{S}_{T}-K\right)^{+} \text {with } \bar{S}_{T}=\frac{1}{n} \sum_{i=1}^{n} S_{i}
$$

a put-call-parity holds. $S_{n}=S_{T}$ and equidistant $S_{i}$ are assumed.

With

$$
\mu=\left(\mathrm{e}^{-\frac{r T(n-1)}{n}}+\cdots+\mathrm{e}^{-\frac{r T}{n}}+1\right)
$$

the following relation is required to avoid arbitrage [7]:

$$
C_{0}=P_{0}+\frac{\mu}{n} S_{0}-K \mathrm{e}^{-r T} .
$$

Variance reduction Reider (1994) [6] suggested to apply put-call-parities to variance reduced importance sampling. In-the-money call/put options can be estimated more efficiently by first estimating the corresponding put/call option with the same parameters and subsequently calculating the required call/put price from the put-call-parity. Conversely, if the approach is applied to out-of-the-money options, the empirical variance is generally increased.

Put-call-parities allow to split a stochastic quantity into deterministic components and a residual stochastic quantity. The deterministic components' standard errors vanish by definition. If the residual stochastic quantity is reduced in absolute size compared to the stochastic quantity of interest, the residual stochastic quantity's standard error will generally tend to be lower as well, all else being equal. According to Gaussian propagation of uncertainty, variance reduction is achieved [7].

\section{Joint Application of the Put-Call-Parity and Importance Sampling}

In a previous article, it is suggested that a combined application of the put-call-parity and importance sampling might be particularly attractive [7]. The author discusses that importance sampling in many cases is especially attractive for out-of-the-money options. Therefore, the valuation of an in-the-money call/put option could be conducted more efficiently by pricing the corresponding out-of-the-money put/call option with importance sampling and then calculating the desired option price from the put-call-parity. More specifically, the following procedure to price call options is examined numerically in the next section:

1) To obtain a benchmark against which variance reductions can be analyzed, a simple Monte-Carlo simulation of the estimator (11) is conducted. Both the estimator and its empirical variance are calculated.

2) To simulate the option price of a call option with a given set of parameters, the corresponding put option with the same set of parameters is simulated using the variance reduced Monte-Carlo estimator (17). $\epsilon$ is calculated using the Black-Scholes formula for put options as described in Equation (18).

3) The call price is calculated from the put-call-parity (20) resp. (23). The empirical variance of this estimated 
call price is the same as the empirical variance of the previously simulated put price. This is the case as all other terms in Equation (20) resp. (23) have zero variance.

4) The variance reduction ratio ("VarRatio") is calculated as the ratio between the empirical variance of the benchmark estimator and the empirical variance of the variance reduced estimator calculated employing importance sampling and the put-call-parity.

\section{Numerical Results}

In this section, we apply the introduced combined approach of importance sampling and employing the putcall-parity to simulate variance reduced Monte-Carlo estimators. To make results comparable, we present two examples that have served previously in the analysis of other approaches discussed in literature [11]. More specifically, we simulate prices of European call options and of arithmetic Asian call options and evaluate achieved variance reductions.

According to the Black-Scholes model, the underlying $S_{t}$ is simulated as follows:

$$
S\left(\tau_{k+1}\right)=S\left(\tau_{k}\right) \exp \left\{\left(r+\epsilon_{k} \sigma^{2}-\frac{\sigma^{2}}{2}\right) \Delta \tau+\sigma \Delta W_{k}\right\} .
$$

We use a discretized time-grid $\tau_{k}=t+k \Delta \tau$ with $n$ equidistant steps.

For benchmark simulations without importance sampling $\epsilon_{k}=0$ follows for all $k$. For simulations involving importance sampling we choose $\epsilon_{k}$ as described in Section 2 .

European options First, we consider the example of a European call option with pay-off function

$$
C_{T}=\left(S_{T}-K\right)^{+}
$$

with $S_{0}=50, r=0.05, T=1.0, n=16$ and $N=10^{6}$ trajectories. For reasons of numerical stability, we truncate the absolute value of the option price elasticity $\epsilon$ to the interval $\left[1 ; 10^{4}\right]$.

Column "PCP-IS method" of Table 1 presents the results of the approach described in section 4. For comparison, results of two other approaches previously discussed in literature are presented as well in the columns "GHS method" and "ZLG method". The first method was presented by Glasserman, Heidelberger and Shahabuddin in 1999 [4] and the second by Zhao, Liu and Gu in 2013 [11]. The numerical results for these two methods are taken from [11].

The approach presented in this article achieves higher variance reductions for all considered parameter constellations compared to the other two approaches. Especially for (deep-)in-the-money options ( $K=30$ ), we observe dramatic variance reductions of several orders of magnitude. For in-the-money options, the approach turns out to be more effective for low-volatility conditions.

Two further aspects should be noted: First, in the case of out-of-the money options a direct application of the introduced importance sampling procedure would be more appropriate than the joint application employing the put-call-parity. E.g., when foregoing the put-call-parity for $K=55$, we obtain variance reduction ratios of 59.3

\begin{tabular}{|c|c|c|c|c|c|c|c|}
\hline \multicolumn{2}{|c|}{ Parameters } & \multicolumn{2}{|c|}{ GHS method } & \multicolumn{2}{|c|}{ ZLG method } & \multicolumn{2}{|c|}{ PCP-IS method } \\
\hline$\sigma$ & $K$ & Price & VarRatio & Price & VarRatio & Price & VarRatio \\
\hline \multirow[t]{4}{*}{0.1} & 30 & 21.463 & 103.3 & 21.463 & 931.2 & 21.463 & 8.37E+17 \\
\hline & 45 & 7.317 & 8.2 & 7.316 & 15.9 & 7.314 & $9.76 \mathrm{E}+03$ \\
\hline & 50 & 3.404 & 7.2 & 3.405 & 12.1 & 3.402 & 201.7 \\
\hline & 55 & 1.087 & 11.2 & 1.088 & 12.5 & 1.086 & 14.5 \\
\hline \multirow[t]{4}{*}{0.3} & 30 & 21.602 & 14.9 & 21.601 & 30.0 & 21.598 & $6.45 \mathrm{E}+04$ \\
\hline & 45 & 9.869 & 9.5 & 9.855 & 15.9 & 9.848 & 283.1 \\
\hline & 50 & 7.118 & 10.3 & 7.121 & 15.8 & 7.115 & 96.6 \\
\hline & 55 & 5.010 & 11.8 & 5.014 & 5.9 & 5.013 & 40.4 \\
\hline
\end{tabular}

Table 1. Estimated variance reduction ratios for European call option. 
for $\sigma=0.1$ resp. 64.2 for $\sigma=0.3$. This is not surprising, as the put-call-parity approach increases empirical variance for out-of-the-money options [7].

Second, the combination of the put-call-parity approach with importance sampling outperforms the two standalone approaches (put-call-parity and importance sampling) for in-the-money options. The standalone importance sampling approach in the case of deep-in-the money calls only leads to limited variance reduction. For the standalone put-call-parity approach applied to the low-volatility case $\sigma=0.1$, we observe that not even one of the $N=10^{6}$ trajectories simulated yields a positive contribution to the Monte-Carlo put option estimator. The result is an empirical variance of zero. Consequently, no reasonable variance reduction can be determined.

Arithmetic Asian options The second example examines an arithmetic Asian option with pay-off function

$$
C_{T}=\left(\bar{S}_{T}-K\right)^{+} \text {with } \bar{S}_{T}=\frac{1}{n} \sum_{i=1}^{n} S_{i} .
$$

Again, we set $S_{0}=50, r=0.05, T=1.0, n=16$ and $N=10^{6}$ trajectories. To approximate the option price elasticity $\epsilon$, the Black-Scholes formula for European options with corresponding parameters has been used. Again, we truncate $\epsilon$. However, in the case of arithmetic Asian options we observe best results for a truncation of its absolute value to the interval $[1 ; 2.5]$.

In the same structure as before, Table 2 shows that we observe the best results for in-the-money options. For low-volatility conditions, we achieve variance reductions of several orders of magnitude. The presented approach outperforms the other methods for in-the-money options. For out-of-the-money options, we achieve no or low variance reduction by employing the combined approach. Again, better results could be achieved by foregoing the put-call-parity.

For European options, we achieved higher variance reduction than for arithmetic Asian options. This is not surprising, as we calculated the option price elasticity $\epsilon$ from the Black-Scholes formula for European options in both examples. Thus, we approximated the optimal importance sampling density more accurately for European options and consequently a higher variance reduction should be expected. More accurate estimations of the option price elasticity for arithmetic Asian options might lead to increased variance reduction ratios.

Again, the numerical results for the GHS method and the ZLG method are taken from [11].

Table 2. Estimated variance reduction ratios for arithmetic Asian call option.

\begin{tabular}{|c|c|c|c|c|c|c|c|}
\hline \multicolumn{2}{|c|}{ Parameters } & \multicolumn{2}{|c|}{ GHS method } & \multicolumn{2}{|c|}{ ZLG method } & \multicolumn{2}{|c|}{ PCP-IS method } \\
\hline$\sigma$ & $K$ & Price & VarRatio & Price & VarRatio & Price & VarRatio \\
\hline \multirow[t]{3}{*}{0.05} & 45 & 6.042 & 39.8 & 6.042 & 931.2 & 6.041 & $2.10 \mathrm{E}+07$ \\
\hline & 50 & 1.437 & 6.4 & 1.438 & 11.5 & 1.438 & 11.5 \\
\hline & 55 & 0.007 & 138.1 & 0.007 & 35.9 & 0.007 & 0.0 \\
\hline \multirow[t]{3}{*}{0.1} & 45 & 6.055 & 10.8 & 6.055 & 24.5 & 6.055 & 754.6 \\
\hline & 50 & 1.919 & 7 & 1.919 & 11.9 & 1.919 & 5.3 \\
\hline & 55 & 0.202 & 21.2 & 0.202 & 13.3 & 0.200 & 0.2 \\
\hline \multirow[t]{3}{*}{0.2} & 45 & 6.418 & 7.7 & 6.419 & 14.5 & 6.419 & 51.2 \\
\hline & 50 & 3.028 & 8.2 & 3.028 & 13.4 & 3.030 & 5.4 \\
\hline & 55 & 1.106 & 13 & 1.106 & 14 & 1.107 & 0.9 \\
\hline \multirow[t]{3}{*}{0.3} & 45 & 7.15 & 8.3 & 7.151 & 15 & 7.150 & 31.2 \\
\hline & 50 & 4.169 & 9.2 & 4.169 & 14.9 & 4.168 & 7.2 \\
\hline & 55 & 2.211 & 12.2 & 2.21 & 15.4 & 2.215 & 2.1 \\
\hline \multirow[t]{3}{*}{0.5} & 45 & 8.996 & 10.6 & 8.996 & 18.6 & 9.001 & 29.3 \\
\hline & 50 & 6.459 & 11.6 & 6.457 & 18.7 & 6.461 & 10.6 \\
\hline & 55 & 4.455 & 13.5 & 4.543 & 18.9 & 4.541 & 4.3 \\
\hline
\end{tabular}




\section{Conclusions}

We conducted variance reduced Monte-Carlo simulations for European and arithmetic Asian options. For inthe-money options, we achieve significant variance reduction by combining importance sampling with the putcall-parity approach. The presented approach delivers best results in low-volatility environments. For European options, it outperforms previously published results for all parameter constellations. Also for arithmetic Asian options, we obtain higher variance reduction ratios for in-the-money options.

This analysis does not consider the combination with other variance reduction techniques, e.g., antithetic variates or stratification, which could lead to further increased variance reduction ratios.

\section{Acknowledgements}

I thank the Editor and the referee for their commitment. I am very grateful to my supervisor Prof. Hermann Singer for the supervision of my research and the related discussions. This work was supported by the Konrad Adenauer Foundation.

\section{References}

[1] Glasserman, P. (2004) Monte Carlo Methods in Financial Engineering. Applications of Mathematics, Vol. 53. Springer, New York, NY; Berlin; Heidelberg.

[2] Melchior, M. and Öttinger, H.C. (1995) Variance Reduced Simulations of Stochastic Differential Equations. The Journal of Chemical Physics, 103, 9506-9509. http://dx.doi.org/10.1063/1.469961

[3] Singer, H. (1999) Finanzmarktökonometrie. Wirtschaftswissenschaftliche Beiträge Nr. 171, Physica-Verl.

[4] Glasserman, P., Heidelberger, P. and Shahabuddin, P. (1999) Asymptotically Optimal Importance Sampling and Stratification for Pricing Path-Dependent Options. Mathematical Finance, 9, 117-152. http://dx.doi.org/10.1111/1467-9965.00065

[5] Singer, H. (2014) Importance Sampling for Kolmogorov Backward Equations. AStA Advances in Statistical Analysis, 98, 345-369. http://dx.doi.org/10.1007/s10182-013-0223-z

[6] Reider, R.L. (1994) Two Applications of Monte Carlo Techniques to Finance. University of Pennsylvania, USA.

[7] Müller, A. (2016) An Application of the Put-Call-Parity to Variance Reduced Monte-Carlo Option Pricing. Fakultät für Wirtschaftswissenschaft, Fern Universität, Hagen, Discussionaper No. 495.

[8] Black, F.and Scholes, M. (1973) The Pricing of Options and Corporate Liabilities. The Journal of Political Economy, 81, 637-654.

[9] Yu, X. and Xie, X. (2013) On Derivations of Black-Scholes Greek Letters. Research Journal of Finance and Accounting, 4, 80-85.

[10] Hull, J.C. (2006) Options, Futures, and Other Derivatives. Pearson Education, Upper Saddle River, NJ, USA.

[11] Zhao, Q., Liu, G. and Gu, G. (2013) Variance Reduction Techniques of Importance Sampling Monte Carlo Methods for Pricing Options. Journal of Mathematical Finance, 3, 431-436. http://dx.doi.org/10.4236/jmf.2013.34045 


\section{Submit or recommend next manuscript to SCIRP and we will provide best service for you:}

Accepting pre-submission inquiries through Email, Facebook, LinkedIn, Twitter, etc.

A wide selection of journals (inclusive of 9 subjects, more than 200 journals)

Providing 24-hour high-quality service

User-friendly online submission system

Fair and swift peer-review system

Efficient typesetting and proofreading procedure

Display of the result of downloads and visits, as well as the number of cited articles

Maximum dissemination of your research work

Submit your manuscript at: http://papersubmission.scirp.org/ 